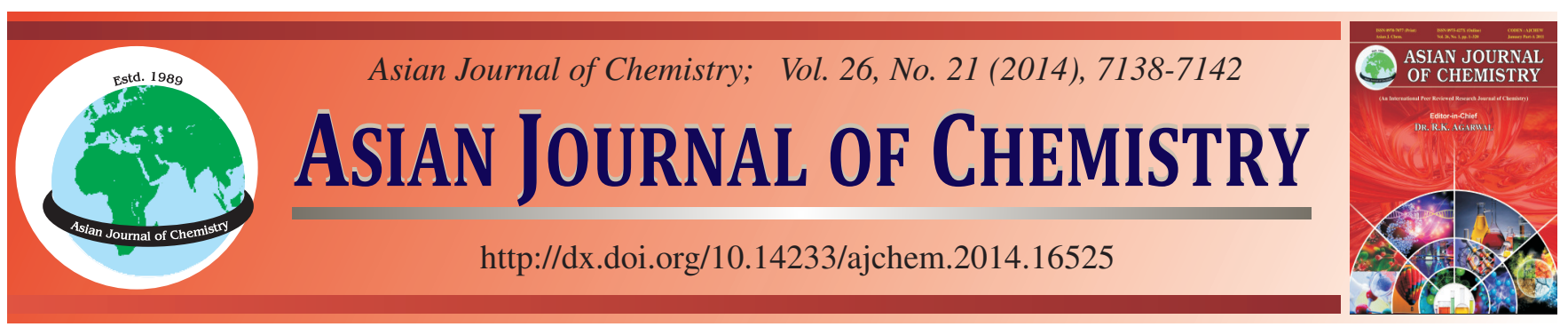

\title{
A Rapid and Sensitive UPLC-MS/MS Method for the Quantification of Danshensu in Dog Plasma and Application to Pharmacokinetics Study
}

\author{
S. CHANG ${ }^{1, *}$, H. Lv ${ }^{2}$, C.R. LIAO ${ }^{3}$ and X.R. ZHANG ${ }^{1, *}$
}

${ }^{1}$ College of Pharmacy, Jilin Medical College, No. 5 Jilin Street, Jilin 132013, P.R. China

${ }^{2}$ Jilin University of Chemical Technology, No. 45, Chengde Street, Jilin 132000, P.R. China

${ }^{3}$ College of Science, Nanchang University, No. 999, Xuefu Road, Nanchang 330031, P.R. China

*Corresponding authors: Tel./Fax: +86 4326456 0532; E-mail: changsheng-pharm@ @otmail.com; yxzxr@163.com

\begin{abstract}
A sensitive, specific and rapid ultra-performance liquid chromatography/tandem mass spectrometry (UPLC-MS/MS) method has been developed and validated for the determination of tanshinol (DSS) in dog plasma in the present study. The analysis were separated on a C18 column $(50 \times 2.1 \mathrm{~mm}, 1.7 \mu \mathrm{m})$ and a triple-quadtriple mass spectrometry equipped with electrospray ionization (ESI) source was applied for detection. The anion exchange solid-phase extraction cartridge was employed to extract the analysis from dog plasma, with high recovery of tanshinol (> $83 \%$ ). The method was linear over the concentration ranges of $1-1000 \mathrm{ng} / \mathrm{mL}$ for tanshinol. The lower limit of quantification (LLOQ) of tanshinol was $1 \mathrm{ng} / \mathrm{mL}$. The intra-day and inter-day relative standard deviation (RSD) were less than $15 \%$ and the relative error (RE) were all within $15 \%$. Finally, the method was successfully applied to support the pharmacokinetic study after the fufang danshen tablet was orally administrated to the beagle dog.
\end{abstract}

Keywords: UPLC-MS/MS, Danshensu, Dog plasma, Solid-phase extraction, Pharmacokinetics.

\section{INTRODUCTION}

Radix Salviae Miltiorrhizae (Danshen in Chinese) was one of the most popular herbs that have been widely applied to promote blood circulation to remove blood stasis, relieve vexation, nourish the blood and cool the blood to relieve carbuncles. Danshensu extracts contained both hydrophilic components [danshensu (DSS, Tanshinol), protocatechuic aldehyde and salvianolic acid B, etc.] and lipophilic components (tanshinone I, tanshinone IIA and cryptotanshinone, etc). Among all these components, plasma and urinary DSS was proved to be a good pharmacokinetic (PK) marker in vivo for some Radix Salviae Miltiorrhizae-contained traditional Chinese medicine by Lu et al. ${ }^{1}$. In the present study, a fufang danshen tablet was developed to cure angina pectoris in coronary heart disease. To evaluate the quality of the preparation, DSS was selected as the PK marker to study the pharmacokinetics in the dog. Therefore, it was indispensable to develop a reliable and facile method to determine the concentration of DSS in dog plasma. Some high-performance liquid chromatography (HPLC) equipped with ultraviolet (UV) and fluorescence (FL) have been developed to determine DSS in dog plasma ${ }^{2}$. But these methods suffered from poor sensitivity and long analysis time. Thus, LC-MS and LC-MS/ MS method was used in the determination of DSS in human and rat plasma with lower limit of quantification (LLOQ) being $2 \mathrm{ng} / \mathrm{mL}^{3-7}$. Up to date, there was no report about the determination of DSS in dog plasma with LC-MS/MS.

This paper described a novel, selective and highly sensitive approach to determine DSS with ultra-performance liquid chromatography coupled with tandem mass spectrometry (UPLC-MS/MS). The developed UPLC-MS/MS method was very sensitive and an anion exchange solid-phase extraction (SPE) for plasma pretreatment generated high recovery and provided much clean samples. The LLOQ of $1 \mathrm{ng} / \mathrm{mL}$ for DSS in the present study corresponded to an on-column sensitivity (the quantity of drug injected on the column per injection) was $1.25 \mathrm{pg}$, which was lower than $3.3 \mathrm{pg}$ recently reported in the literature ${ }^{7}$. The analysis time per injection was only $4 \mathrm{~min}$. The combination of high sensitivity with rapid analysis made this method suitable for the high throughput screening. The method was then validated and successfully applied to the PK study after the fufang danshen pulsatile table was orally administrated to the beagle dog.

\section{EXPERIMENTAL}

DSS (> $99 \%$ purity) and $p$-hydroxybenzoic acid (IS, > $99 \%$ purity) was purchased from the National Institute for Control of Pharmaceutical and Biological Products (Beijing, 
China). Acetonitrile, methanol and formic acid (HPLC grade) were purchased from Dikma (Richmond Hill, NY, USA). Water was purified using a Milli-Q system (Millipore Corporation, Billerica, MA). Oasis MAX sorbents (30 mg/1cc) were provided by Waters Corp. (Milford, MA, USA).

A Waters Acquity UPLC system coupled with a triple quadrupole tandem mass spectrometer (Micromass Quattro Premier XE mass spectrometer, Waters Corp., Milford, MA, USA) with electrospray ionization (ESI) interface was employed for analysis. An UPLC BEH C18 column $(50 \times 2.1$ mm, 1.7 um, waters Corp., Milford, MA, USA) was used to separate the analytes. All data were collected in centroid mode and processed using MassLynxTM NT 4.0 software with a QuanL ynxTM program (Waters Corp., Milford, MA, USA).

UPLC/MS/MS conditions: A gradient elution program was conducted for chromatographic separation with the mobile phase $\mathrm{A}$ (acetonitrile) and the mobile phase $\mathrm{B}$ (water containing $0.5 \%$ formic acid) as follows: $0 \mathrm{~min}(5 \%, \mathrm{~A}), 2.5 \mathrm{~min}(30 \%$, A), $2.51 \mathrm{~min}(5 \%, \mathrm{~A}), 4 \mathrm{~min}(5 \%, \mathrm{~A})$ and finished at $4 \mathrm{~min}$. The flow rate was $0.15 \mathrm{~mL} / \mathrm{min}$ and column temperature was $40{ }^{\circ} \mathrm{C}$. Injection wash solvents were methanol-water- $0.1 \%$ formic acid (5:95:0.1, v/v/v) and methanol-water- $0.1 \%$ formic acid (95:5:0.1, v/v/v) for weak and strong wash, respectively. For MS detection, negative ESI was used as the ionization mode. Nitrogen was used as the desolvation and cone gas with a flow rate of 600 and $50 \mathrm{~L} / \mathrm{h}$, respectively. High purity argon was used as the collision gas at a pressure of approximately $3.05 \times 10^{-3}$ mbar. The optimal MS parameters were as follows: capillary $2.5 \mathrm{kV}$, source temperature $120^{\circ} \mathrm{C}$ and desolvation temperature $380{ }^{\circ} \mathrm{C}$. Cone voltage was $22 \mathrm{~V}$ for both DSS and p-hydroxybenzoic acid. Quantification was performed using multiple reaction monitoring (MRM) of the transitions of $\mathrm{m} / \mathrm{z}$ $197 \rightarrow 135$ with collision energy (CE) of $16 \mathrm{eV}$ for DSS and $m / z 137 \rightarrow 93$ with $\mathrm{CE}$ of $20 \mathrm{eV}$ for $p$-hydroxybenzoic acid.

Preparation of standard and quality control samples: Standard stock solution of DSS was prepared in water at the concentration of $0.4 \mathrm{mg} / \mathrm{mL}$. The working solution of internal standard $(1 \mu \mathrm{g} / \mathrm{mL})$ was also prepared in water. A series of standard solutions of $1,2,5,50,100,500,1000 \mathrm{ng} / \mathrm{mL}$ for DSS was prepared by serially diluting the stock solution. All the solutions were stored at $4{ }^{\circ} \mathrm{C}$. DSS calibration curves were prepared by spiking $50 \mathrm{~mL}$ of blank rat plasma with $50 \mu \mathrm{L}$ of DSS standard solution, $50 \mu \mathrm{L}$ of internal standard solution. The quality control (QCs) samples at low, medium, high concentration levels were prepared in the same way as the DSS calibration curves. The nominal plasma concentrations of QC samples were 2, 500, $800 \mathrm{ng} / \mathrm{mL}$, respectively.

Sample preparation: To $50 \mu \mathrm{L}$ aliquot of plasma sample, $50-\mu \mathrm{L}$ of internal standard solution $(1 \mu \mathrm{g} / \mathrm{mL}), 50 \mu \mathrm{L}$ of water, $10 \mu \mathrm{L}$ of hydrochloric acid solution (1 M) was added. After briefly mixing for $1 \mathrm{~min}$, the mixture was loaded on a solidphase extraction cartridge which had been pre-activated and equilibrated with $2 \mathrm{~mL}$ methanol and then $2 \mathrm{~mL}$ water before use. The sample was washed by $2 \mathrm{~mL} 5 \%$ ammonium hydroxide solution and then by $2 \mathrm{~mL}$ methanol. DSS and $p$ hydroxybenzoic acid were eluted with $2 \mathrm{~mL}$ methanol containing $2 \%$ formic acid. The eluates were evaporated under a stream of nitrogen at $37^{\circ} \mathrm{C}$ and the residue was reconstituted in $200 \mu \mathrm{L}$ acetonitrile-water (50:50) by vortex mixing (1 min).
The resulting solution was transferred to an autosampler vial at $4{ }^{\circ} \mathrm{C}$ and injected $(5 \mu \mathrm{L})$ into UPLC/MS/MS system for analysis. The plasma was diluted with the blank plasma if the concentration of DSS in plasma was above the range of the linearity.

Method validation: To determine whether endogenous constituents interfere with analytes quantification, blank dog plasma samples from six different donors and LLOQ samples were pretreated and analyzed. LLOQ was determined with acceptable precision and accuracy [six replicates on three validation days within a relative standard deviation (RSD) below $20 \%$ and a relative error (RE) within $\pm 20 \%$ ]. Linearity of calibration curves based on peak area ratio (area of DSS/ area of IS) (y) as function of the nominal concentration (x) was calculated by weighted $(1 / \mathrm{x} 2)$ least square regression and was expressed in the form $y=a+b x$. Intra-day and inter-day precision (the relative standard deviation, RSD) and accuracy (the relative error, RE) were determined by analysis of low, medium and high QC samples $(n=6)$ on three consecutive days. The matrix effect was investigated by comparing the peak areas of analytes in the post-extraction spiked blank plasma at low and high concentrations with those of the corresponding standard solutions. The extraction recovery was determined by comparing the mean peak areas of six extracted samples at low, medium and high QC concentrations with the mean peak areas of spike-after-extraction samples. The stability of low and high QC samples $(\mathrm{n}=3)$ in three complete freeze/ thawcycles $\left(-80\right.$ to $\left.22{ }^{\circ} \mathrm{C}\right)$, long-term sample storage $\left(-80{ }^{\circ} \mathrm{C}\right.$ for 30 days $)$ and bench-top $\left(23^{\circ} \mathrm{C}\right.$ for $2 \mathrm{~h}$ ) was assessed. The ready to-injection stability of extracted samples in the autosampler rack at $4{ }^{\circ} \mathrm{C}$ for $24 \mathrm{~h}$ was also evaluated.

Pharmacokinetic (PK) study in beagle dogs: The validated method was applied to determine the concentration of DSS in dog plasma after an oral administration of the fufang danshen tablet ( $5 \mathrm{mg} / \mathrm{kg}$ calculated as DSS) to six healthy beagle dogs. All animal experiments were performed in accordance with institutional guidelines and were approved by the Committee on Use and Care of Animals, Jilin Medical College. The dogs were $(12-15 \mathrm{~kg}$ ) were fasted for $12 \mathrm{~h}$ prior to the experiment but free access to water. Serial venous blood samples $(10 \mathrm{~mL})$ were obtained at $0,0.5 \mathrm{~h}$ and $1,2,4,5,6,8$, $10,12,24 \mathrm{~h}$ after oral administration. After centrifugation at $3000 \times \mathrm{g}$ and $4{ }^{\circ} \mathrm{C}$ for $10 \mathrm{~min}$, plasma was collected and frozen at $-80{ }^{\circ} \mathrm{C}$ until analysis.

Data analysis: To determine the pharmacokinetics parameters of DSS, all data were processed by non-compartmental analysis using the DAS 2.0 software package (Chinese Pharmacological Society). The plasma concentration at different times was expressed as mean \pm standard deviation (S.D.) and the mean concentration-time curves were plotted. The maximum plasma concentration $\left(\mathrm{C}_{\max }\right)$ and the time $\left(\mathrm{t}_{\max }\right)$ to reach it were observed directly from the concentration-time curves. The area under the plasma concentration-time curve from zero to the time of the final measurable sample $\left(\mathrm{AUC}_{0-\mathrm{t}}\right)$ was calculated using the linear-trapezoidal rule up to the last sampling point with the detectable level (C). The area under the plasma concentration-time curve from zero to infinity $\left(\mathrm{AUC}_{0-\infty}\right)$ was calculated using the linear-trapezoidal rule with extrapolation to infinity. 


\section{RESULTS AND DISCUSSION}

Optimization of method: DSS had a carboxyl group in the molecular structure. It has a stronger mass response under the negative ionization mode than the positive mode. It was the same case for the internal standard $p$-hydroxybenzoic acid. In addition, ESI source provided a better response than APCI source for two analytes. The product spectrum of the $[\mathrm{M}-\mathrm{H}]$ of DSS was dependent on the collision energy. The major fragment ion $\mathrm{m} / \mathrm{z} 135$ was formed at higher collision energy $(16 \mathrm{eV})$ and the most abundant ion $\mathrm{m} / \mathrm{z}, 179$ was formed at lower collision energy $(11 \mathrm{eV})$. The transition of $\mathrm{m} / \mathrm{z}, 197 \rightarrow$ 135 gave a higher signal-to-noise $(\mathrm{S} / \mathrm{N})$ ratio and better response than that of $\mathrm{m} / \mathrm{z} 197 \rightarrow 179$ during the analysis of the spiked plasma samples. As a result, the transition of $m / z$ $197 \rightarrow 135$ was selected for MRM analysis. In the same way, the transition of $m / z, 137 \rightarrow 93$ for $p$-hydroxybenzoic acid was selected for MRM analysis (Fig. 1). Chromatographic conditions were optimized to obtain better peak shape and higher sensitivity. The addition of $0.5 \%$ formic acid to the mobile phase could improve the shape of DSS and did not
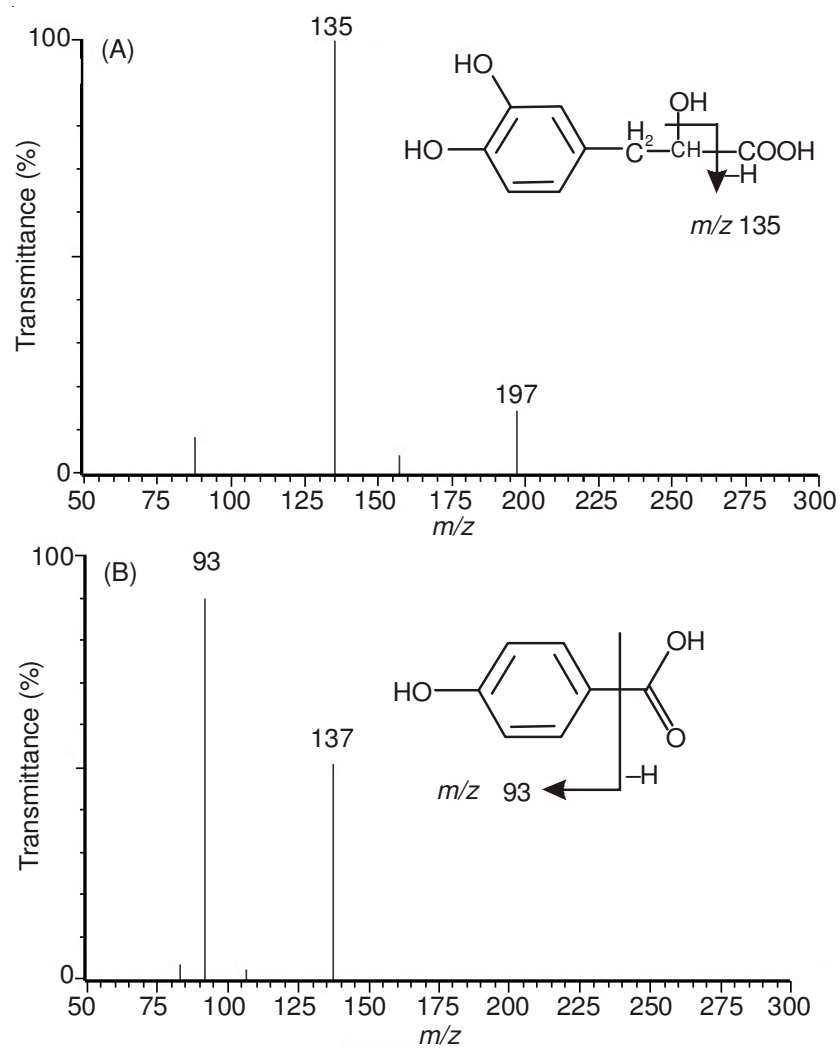

Fig. 1. Production ion mass spectra of $[\mathrm{M}-\mathrm{H}]^{-}$of DSS (A) and $p$-hydroxybenzoic acid (B) decrease the mass response of the analytes. The protein precipitation using methanol or acetonitrile and the liquidliquid extraction pretreatment were employed to extract DSS from plasma in the previous reports. But during our exploration, we found that these methods resulted in a very low extraction recovery for DSS and the interference from the endogenous matrix was observed. Because DSS and $p$ hydroxybenzoic acid are acidic, anion exchange solid-phase extraction cartridges were applied for their extraction, which gave recovery above $83 \%$ for all analytes. Furthermore, this solid-phase extraction procedure can provide cleaner samples than the other pretreatment methods, thus reduced the background noise and increased the sensitivity. So it was selected as plasma pretreatment method.

\section{Method validation}

Selectivity: Fig. 2 shows the typical chromatograms of a blank, a spiked plasma sample with DDS (1 ng/mL) and the internal standard $(1 \mu \mathrm{g} / \mathrm{mL})$, a plasma sample from a dog after an oral administration of fufang danshen pulsatile tablet. No interference from the endogenous matrix with the analytes and the internal standard was detected.

Linearity of calibration curves and LLOQ: Calibration curves were obtained between the mass responses and the plasma concentration over the range of $1-1,000 \mathrm{ng} / \mathrm{mL}$ for DDS as described above. The typical regression equation was as follows: $\mathrm{y}=0.00593 \times+0.00657(\mathrm{r}=0.996)$. All correlation coefficients (r) exceeded 0.99 , showing a good linearity over the concentration range. The LLOQ was $1 \mathrm{ng} / \mathrm{mL}$ for DSS in dog plasma. The intra- and inter-RSD were both less than $8.9 \%$ and the relative error were within $9.6 \%$ at LLOQ level, which were within the acceptable limits.

Precision and accuracy: The intra-day and inter-day precision and accuracy for DSS at three QC concentration levels were shown in Table-1. The intra-day and inter-day precision values were from 3.8 to $6 \%$. The accuracy ranged from 5 to $7.8 \%$. The results indicated that all the values were within the acceptable range and the method showed good reproducibility and accuracy.

Extraction recovery: The mean extraction recovery was $83.1 \pm 3.4,85.7 \pm 2.8$ and $89.1 \pm 4.5 \%$ at 2,500 and $800 \mathrm{ng} / \mathrm{mL}$, respectively. The mean recovery of the internal standard was $88.4 \pm 4.8 \%$. All recoveries had RSD less than $5 \%$ at three different concentrations, showing good assay consistency.

Matrix effects: The matrix effects calculated were in the range of -7.1 to $4.5 \%$, which was within the acceptable limits. Therefore, there was no significant matrix effects observed in this method.

Stability: Stability results in Table- 2 demonstrated that DSS was stable in dog plasma under the indicated conditions.

TABLE-1

ACCURACY AND PRECISION FOR THE ANANLYSIS OF DDS IN RAT PLASMA (IN THREE VALIDATION, SIX REPLICATES AT EACH CONCENTRATION LEVEL PER DAY)

\begin{tabular}{ccccc}
\hline \multicolumn{2}{c}{ Concentration $(\mathrm{ng} / \mathrm{mL})$} & \multicolumn{2}{c}{ RSD $(\%)$} & Ralative error $(\%)$ \\
\hline Added & Found(mean) & Intra-day & Inter-day & \\
\hline 2 & 2.1 & 4.5 & 5.3 & 5.0 \\
500 & 539.0 & 5.9 & 3.8 & 7.8 \\
800 & 844.0 & 6.0 & 4.2 & 5.5 \\
\hline
\end{tabular}



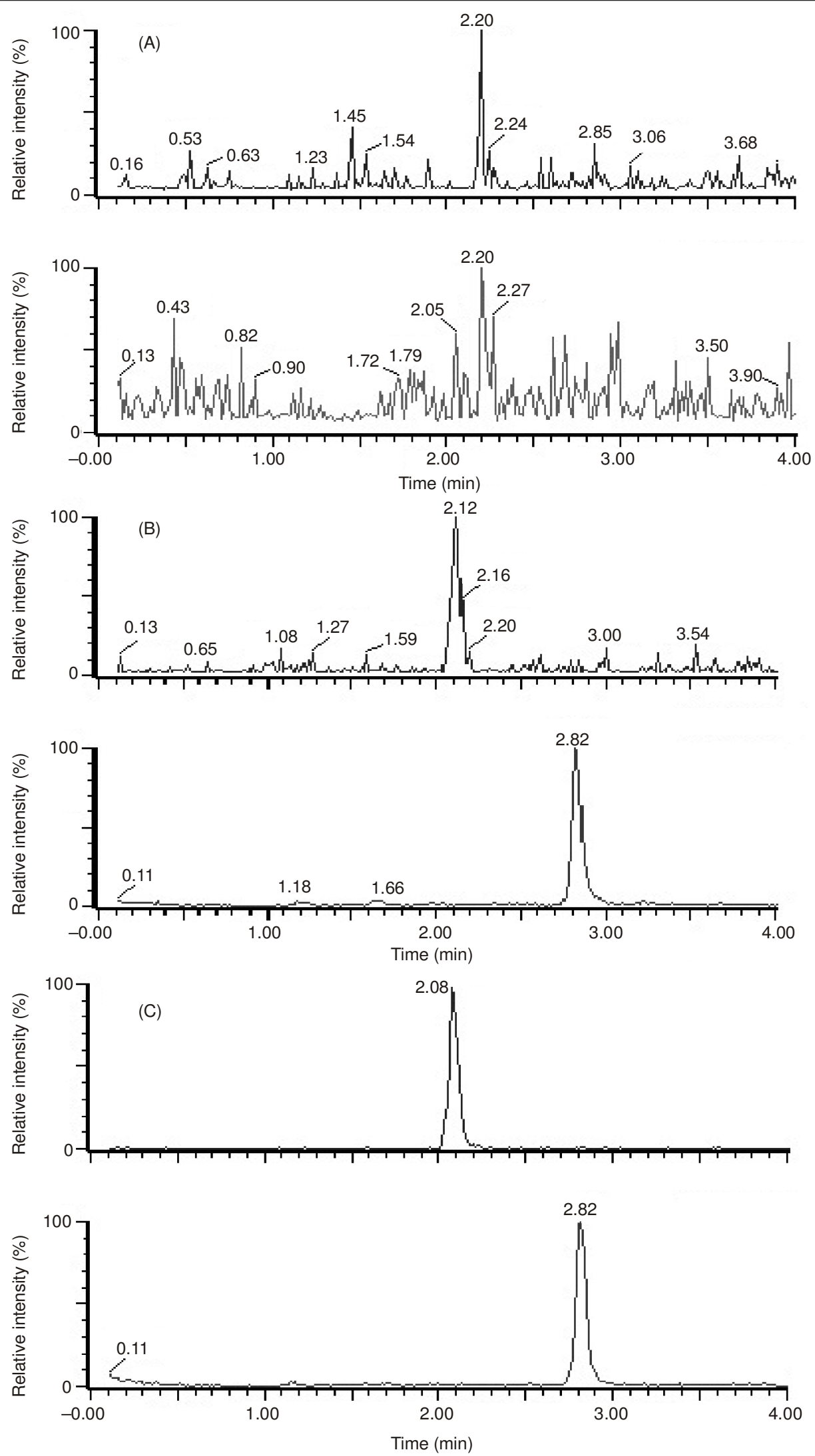

Fig. 2. Representative MRM chromatograms of DDS and p-hydroxybenzcic acid (IS) in dog plasmas: (A) a blank dog plasma sample; (B) a blank dog plasma sample spiked with DSS $(1 \mathrm{ng} / \mathrm{mL})$ and $p$-hydroxybenzcic acid $(1 \mu \mathrm{g} / \mathrm{mL}) ;(C)$ a plasma sample following an oral dose of fufang danshen pulsatile tablet to the Beagle dogs

Application to PK study in Beagle dog: This developed and validated analytical method was successfully applied to the evaluation of the PK behaviour of DSS in dogs after an oral administration of fufang danshen tablet to the Beagle dog. The typical plasma concentration-time profile of DSS was shown in Fig. 3. The $\mathrm{T}_{\max }$ and $\mathrm{C}_{\max }$ were observed from Fig. 3 
TABLE-2

STABILITY DATA OF DSS IN DOG PLASMA UNDER DIFFERENT CONDITIONS

\begin{tabular}{|c|c|c|c|c|}
\hline \multirow{2}{*}{ Storage conditions } & \multicolumn{4}{|c|}{ Concentration (ng/mL) } \\
\hline & Added & Found & $\operatorname{RSD}(\%)$ & Relative error (\%) \\
\hline \multirow{2}{*}{ Bench-top $2 \mathrm{~h}$} & 2 & 1.9 & 4.3 & -5.0 \\
\hline & 800 & 771.2 & 5.2 & -3.6 \\
\hline \multirow{2}{*}{ Three freeze/thaw cycles } & 2 & 2.1 & 3.8 & 5.1 \\
\hline & 800 & 857.6 & 5.7 & 7.2 \\
\hline \multirow{2}{*}{ Autosampler rack for $24 \mathrm{~h}{ }^{\circ} \mathrm{C}$} & 2 & 1.8 & 6.2 & -10.1 \\
\hline & 800 & 838.4 & 4.9 & 4.8 \\
\hline \multirow{2}{*}{ Freezing for $30 \mathrm{~d}$ at $-80^{\circ} \mathrm{C}$} & 2 & 2.2 & 8.1 & 9.6 \\
\hline & 800 & 848.0 & 3.2 & 6.0 \\
\hline
\end{tabular}

and the results were $1 \mathrm{~h}$ and $3398.4 \mathrm{ng} / \mathrm{mL}$, respectively. Plasma concentration declined with $\mathrm{t}^{1 / 2} 5.9 \pm 1.5 \mathrm{~h}$. $\mathrm{AUC}_{0-24} \mathrm{~h}$ and $\mathrm{AUC}_{0-\infty}$ values were 15839.4 and 17531.9 ( $\left.\mathrm{ng} \mathrm{h} / \mathrm{mL}\right)$, respectively. Fig. 3 showed that the absorption was very fast.

\section{Conclusion}

A sensitive, specific and rapid UPLC-MS/MS method was developed for the analysis of DSS in dog plasma. The anion

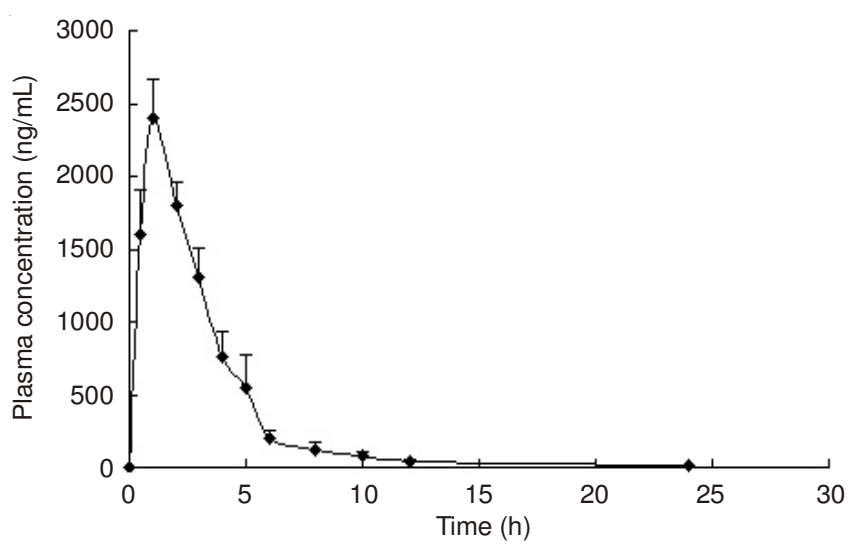

Fig. 3. Plasma concentration-time profiles of DSS in the Beagle dogs following oral administration of fufang danshen pulsatile tablet (5 $\mathrm{mg} / \mathrm{kg}$ calculated as DSS) to the Beagle dog exchange solid phase extraction procedure resulted in high extraction recovery for DSS and clean sample for analysis. The LLOQ of thepresent method was $1 \mathrm{ng} / \mathrm{mL}$, which was lower than the LLOQ recently reported in the literature. The method has been successfully applied to the PK study of DSS after oral dosing of fufang danshen tablet to the beagle dogs.

\section{REFERENCES}

1. T. Lu, J.L. Yang, X.M. Gao, P. Chen, F. Du, Y. Sun, F. Wang, F. Xu, H. Shang, Y. Huang, Y. Wang, R. Wan, C. Liu, B. Zhang and C. Li, Drug Metab. Dispos., 36, 1578 (2008).

2. X.J. Luo, K.S. Bi, S.Y.Zhou, Q. Wei and R. Zhang, Biomed. Chromatogr., 15, 493 (2001).

3. B.B. Chang, L. Zhang, W.W. Cao, Y. Cao, W. Yang, Y. Wang, Y. Chen and X. Liu, Acta Pharmacol. Sin., 31, 638 (2010).

4. X.R. Guo, X.H. Chen, L. Li, Z. Shen, X. Wang, P. Zheng, F. Duan, Y. $\mathrm{Ma}$ and K. Bi, J. Chromatogr. B, 873, 51 (2008).

5. X.H. Li, C. Yu, Y.B. Cai, G. Liu, J. Jia and Y. Wang, J. Chromatogr. B, 820, 41 (2005).

6. Y. Liu, X.R. Li, Y.H. Li, L. Wang and M. Xue, J. Pharm. Biomed. Anal., 53, 698 (2010).

7. S.P. Wang, L. Liu, L.L. Wang, P. Jiang, L. Xiang, W.-D. Zhang and R.-H. Liu, J. Chromatogr. B, 912, 75 (2013). 